\title{
La Cour de Justice dans les négociations du traité de Paris instituant la CECA
}

\author{
Anne BOERGER-DE SMEDT
}

Le rôle extraordinaire joué par la Cour de Justice européenne (CJE) dans le processus d'intégration n'est aujourd'hui plus à démontrer. Un nombre impressionnant de publications ont d'ores et déjà mis en exergue la véritable révolution juridique que la Cour a imposée par voie d'une jurisprudence basée sur une interprétation hardie des traités. Juristes, politologues et, plus récemment, historiens, ont montré à foison comment certains arrêts audacieux des juges de Luxembourg ont favorisé l'avancement de l'unification européenne, notamment en procédant à une sorte de «constitutionnalisation» des traités fondateurs. Ces travaux se concentrent essentiellement sur la période postérieure à l'entrée en vigueur des traités de Rome et ce, non sans raison puisque c'est dans le cadre de la CEE, après qu' une Cour de justice unique ait remplacé celle de la CECA, que les arrêts les plus décisifs et spectaculaires ont été rendus, notamment ceux affirmant la primauté et l'effet direct du droit communautaire. $^{1}$

Par contraste avec cette abondante littérature, peu d'auteurs ont porté leur regard en amont des traités de Rome et moins encore se sont efforcés de comprendre comment et à quels fins un organe judiciaire a émergé lors des négociations du traité de Paris avant d'évoluer durant les tractations en vue de la création de la CEE. L'histoire de l'intégration juridique de l'Europe ne pourrait pourtant être complète - ni pleinement intelligible - si l'on néglige la genèse de la CJE et les développements initiaux du droit communautaire. ${ }^{2}$ Le présent article ambitionne de combler, si imparfaitement soit-il, certaines de ces lacunes en analysant dans les détails les circonstances et les idées qui ont présidé à la conception de la première cour européenne, celle de la CECA. ${ }^{3}$

On dispose certes déjà de divers écrits sur la gestation de la Cour durant la conférence de Paris, écrits que l'on doit soit à la plume de quelques juristes et autres négociateurs directement impliqués dans cette phase créatrice, soit à celle des historiens des débuts de la construction européenne. Ces publications sont utiles mais présentent certaines limites que nous espérons dépasser par nos

1. Je tiens à remercier Morten Rasmussen et Piers Ludlow pour leurs commentaires.

2. Sur le développement du droit communautaire entre 1953 et 1958, voir M. LAGRANGE, Le rôle de la Cour de Justice des Communautés européennes tel qu'il se dégage de sa jurisprudence, in: Droit social, 1(1961), pp.1-9; Idem. The Court of Justice as a Factor in European Integration, in: The American Journal of Comparative Law, 4(1966/67), pp.709-725; C. PENNERA, The Beginnings of the Court of Justice and its Role as a driving Force in European Integration, in: Journal of European Integration History, 1(1995), pp.111-127; S.A. SCHEINGOLD, The Rule of Law in European Integration - The Path of the Schuman Plan, Yale University Press, New Haven-Londres, 1965.

3. Un second article, consacré à la CJE durant les négociations des traités de Rome, viendra compléter la présente étude. 
recherches. Parmi les juristes qui ont relaté leur expérience à la conférence de Paris et/ou analysé par la suite le fonctionnement institutionnel de la CECA, quelques noms s'imposent, dont celui de Paul Reuter, ${ }^{4}$ ce professeur de droit à l'Université d'Aix-en-Provence qui collabora à l'élaboration du Plan Schuman dès avril 1950, celui du juriste allemand, Hermann Mosler, ${ }^{5}$ ou encore celui d'André Gros, ${ }^{6}$ jurisconsulte au ministère français des Affaires étrangères. Le magistrat le plus souvent associé à la création de la Cour européenne reste cependant Maurice Lagrange. ${ }^{7}$ Recruté à la demande de Monnet, ce membre du Conseil d'État rejoignit l'équipe de la rue de Martignac au début de l'automne 1950. Fort de son expérience du droit administratif français, il joua un rôle clé dans la rédaction des articles relatifs à la Cour et dans la définition de ses compétences. Lagrange poursuivit sa carrière européenne en devenant un des deux premiers avocats généraux à la CJE, fonction qu'il assuma jusqu'en 1964. Précieux, son témoignage s'est imposé au fil des années comme le récit le plus fiable de la genèse de la Cour et a largement contribué à asseoir l'idée que ce fut grâce à son intervention que l'organe judiciaire de la CECA fut pratiquement une copie du Conseil d'État français. Reprise par de nombreux auteurs comme une vérité établie, ${ }^{8}$ cette affirmation relève pourtant davantage du mythe que de la réalité historique, ainsi que nous aurons l'occasion de le démontrer à la lumière de documents d'archives. Outre ces témoins privilégiés, plusieurs historiens des débuts de la construction européenne ont également retracé les origines du Plan Schuman et relaté les

4. P. REUTER, La conception du pouvoir politique dans le Plan Schuman, in: Revue française de science politique, 1(1951), pp.256-276; Idem., Quelques aspects institutionnels du Plan Schuman, in: Revue de droit public et de la science politique en France et à l'étranger, 1951, pp.105-124; Idem., La Communauté Européenne du Charbon et de l'acier, Institut des relations internationales, Paris, 1953; Idem., Juridical and Institutional Aspects of the European Regional Communities, in: Law and Contemporary Problems,3(1961/62), pp.381-399; Idem., Aux origines du Plan Schuman, in: Mélanges Fernand Dehousse, vol.2 La construction européenne, Labor, Bruxelles, 1979, pp.65-68. Sur Reuter, voir A. COHEN, Le plan Schuman de Paul Reuter. Entre communauté nationale et fédération européenne, in: Revue française de science politique, 5(1998), pp.645-663.

5. H. MOSLER, Der Vertrag über die Europäische Gemeinschaft für Kohle und Stahl. Entstehung und Qualifizierung, in: Zeitschrift für ausländisches öffentliches Recht und Völkerrecht, 14(1951/ 52), pp.1-45; Idem., Die Entstehung des Modells supranationaler und gewaltenteilender Staatenverbindungen in den Verhandlungen über den Schuman-Plan, in: E. von CAEMMERER (dir.), Probleme des Europäischen Rechts. Festschrift für Walter Hallstein, Klostermann, Frankfurt a.M., 1966, pp.355-386.

6. Gros a peu écrit sur la CECA. Durant les négociations, il évoqua la cour de la CECA dans A. GROS, The Problem of Redress against the Decisions of International Organisations, in: Transactions of the Grotius Society, 36(1950), pp.29-48.

7. Outre les articles déjà mentionnés, voir M. LAGRANGE, L'ordre juridique de la CECA vu à travers la jurisprudence de sa Cour de Justice, in: Revue du droit public et de la science politique en France et à l'étranger, 74(1958), pp.841-865; Idem., La Cour de Justice des Communautés européennes du Plan Schuman à l'Union européenne, in: Mélanges Fernand Dehousse ..., op.cit., pp. 127-135. Voir aussi Fondation Jean Monnet (FJM), Interview de M. Lagrange par A. Marès, 23.09.1980.

8. Cf. par exemple J. MERTENS DE WILMARS, La contribution des juristes belges à l'intégration européenne, in: Studia Diplomatica, xxxiv(1981), p.141; J. WILSON, Aux origines de l'ordre juridique communautaire, in: C. FRANCK et S. BOLDRINI, Une constitution pour un projet et des valeurs, Bruylant, Bruxelles, 2004, pp.22-23. 
négociations du traité CECA. Certains de leurs travaux, comme ceux de Pierre Gerbert et de Raymond Poidevin, proposent une analyse de l'ensemble de la conférence de Paris; ${ }^{9}$ d'autres s'intéressent davantage à la position de telle ou telle délégation durant ces pourparlers; ${ }^{10}$ Hanns-Jürgen Küsters a quant à lui consacré un article à la genèse du système institutionnel durant cette phase créatrice. ${ }^{11}$ Aucun de ces auteurs ne prête toutefois une attention particulière à la Cour de Justice.

Pour nuancer les souvenirs parfois biaisés des témoins, et afin de compléter les travaux des historiens, une recherche extensive dans les archives des pays fondateurs et dans des fonds américains s'imposait. Nous avons entrepris cet examen et nous nous proposons maintenant d'expliquer, à la lumière de documents historiques, la genèse de l'organe judiciaire de la CECA. La première partie de l'article, consacrée à la période incluse entre la préparation de la déclaration du 9 mai et le début du mois d'août 1950, expose les nombreux détours par lesquels les négociateurs sont passés avant de conclure que la future communauté européenne requérait une juridiction propre, unique et permanente. Ces trois caractéristiques semblent aujourd'hui aller de soi; elles n'émergèrent pourtant que progressivement - et difficilement - de projets initiaux toujours très marqués par les principes de l'organisation judiciaire internationale classique. La seconde partie de l'étude relate les intenses discussions qui, essentiellement à l'automne 1950, permirent aux juristes de définir la composition et les compétences de la Cour. Puisqu'il serait trop ambitieux de passer en revue toutes ses caractéristiques, nous nous limiterons à celles qui suscitèrent le plus de difficultés durant la conférence de Paris. Soulignons enfin que notre approche se veut avant tout historique: au-delà de la technique juridique, nous nous efforçons donc d'expliquer les facteurs politiques, les influences académiques, les négociateurs clés qui, directement ou indirectement, contribuèrent à façonner la première cour européenne.

9. P. GERBERT, La genèse du Plan Schuman, in: Revue Française de Science Politique, 3(1956), pp.525-553; Idem., La genèse du Plan Schuman. Des origines à la déclaration du 9 mai 1950, Sciences Po, Lausanne, 1962; R. POIDEVIN, D.SPIERENBURG, Histoire de la Haute Autorité de la Communauté Européenne du Charbon et de l'Acier. Une expérience supranationale, Bruylant, Bruxelles, 1993, pp.9-52.

10. Voir les contributions de M. DUMOULIN et A. KERSTEN in: K.SCHWABE (éd.), Die Anfänge des Schuman-Plans 1950/1951, Nomos, Baden-Baden, 1988; R.T. GRIFFITHS, Die Benelux-Staaten und die Schumanplan-Verhandlungen, in: L. HERBST(éd.), Vom Marshallplan zur EWG. Die Eingliederung der Bundesrepublik Deutschland in die Westliche Welt, Ôldenbourg, Munich, 1990, pp.263-278; H-J. KÜSTERS, Bonn und der Schumanplan. Deutsche Europapolitik in den Verhandlungen über die Montanunion 1950/1951, in: Geschichte im Westen, 5(1990), pp.81-100; R. RANIERI, Italy and the Schuman Plan Negotiations, IUE, Florence, 1986; G. TRAUSCH, Le Luxembourg face au plan Schuman: la place d'un petit pays dans la construction européenne, in: M. CATALA (dir.), Histoire de la construction européenne. Cinquante ans après la Déclaration Schuman, Ouest éditions, Nantes, 2001, pp.195-224.

11. H.-J. KÜSTERS, Die Verhandlungen über das institutionelle System zur Gründung der Europäischen Gemeinschaft für Kohle und Stahl, in: K. SCHWABE (éd.), Die Anfänge ..., op.cit., pp.73-102. 


\section{Des «dispositions appropriées» à une Cour de Justice unique, permanente et propre à la CECA}

La déclaration du 9 mai 1950, aujourd'hui considérée comme l'acte de naissance de l'Union européenne, ne contenait qu'un bref paragraphe sur les institutions à créer pour permettre la mise en œuvre de l'initiative lancée par Robert Schuman. Ce paragraphe est passé à la postérité car il annonçait la création de la Haute Autorité supranationale, un organe inédit sur lequel devait reposer le succès du plan proposé. Outre cette pièce maîtresse de l'édifice institutionnel, l'alinéa en question mentionnait aussi que «des dispositions appropriées assureront les voies de recours nécessaires contre les décisions de la Haute Autorité». Le 9 mai 1950 pourtant, les auteurs du célèbre texte n'avaient encore aucune idée précise de ce qu'allaient être ces «dispositions appropriées». À ce stade, l'organisation des recours n'implique certainement pas la création d'une nouvelle cour de justice. En fait, la nécessité même d'organiser un recours contre les décisions du nouvel organe supranational ne s'était pas immédiatement imposée à Jean Monnet et aux trois experts (Paul Reuter, Étienne Hirsch et Pierre Uri) qui l'ont assisté dans l'élaboration de la déclaration Schuman puisque le principe ne fut introduit qu'à l'occasion de la correction de la cinquième version du texte. Ajoutée au passage consacré à la Haute Autorité, une phrase précisa alors - et ce pour la première fois -, que les décisions du collège supranational «ne seront révisables que par appel devant la Cour permanente de Justice Internationale». ${ }^{12}$ Doutant ensuite que la Cour de La Haye constituerait l'instance la mieux qualifiée pour accomplir une telle tâche, ${ }^{13}$ les auteurs du Plan Schuman préférèrent écarter cette solution lors de la rédaction de la huitième version de la déclaration et optèrent pour la formule plus vague citée ci-dessus.

Durant les six semaines comprises entre le lancement du Plan Schuman et l'ouverture officielle de la conférence de Paris (21 juin 1950), Reuter et Uri se prêtèrent à un intense exercice d'imagination juridique destiné à préciser la structure institutionnelle nécessaire au fonctionnement du futur pool charbon-acier. La Haute Autorité, pierre angulaire de l'échafaudage institutionnel, retint naturellement l'essentiel de leur attention, mais la question des recours fut aussi l'objet de sérieuses réflexions. Le sujet était complexe puisqu'il s'agissait notamment de déterminer devant quelle(s) instance(s) un appel pourrait être introduit, par qui et pour quels motifs. Initialement, Uri et Reuter identifièrent trois types de contentieux et prévirent en conséquence trois instances arbitrales. D'abord, la Haute Autorité elle-même devait faire office d'organe de première instance: ses membres procéderaient à une seconde lecture d'une décision contestée et, si nécessaire, la confirmeraient à une majorité des deux

12. FJM, AMG $1 / 2,5^{\text {ème }}, 6^{\text {ème }}$ et $7^{\text {ème }}$ versions de la déclaration Schuman.

13. FJM, AMG 2/3/8, Haut-commissariat de la République de France en Allemagne. Exposé de M. Monnet devant le Conseil de la Haute Commission Alliée, Petersbourg, 23 mai 1950, Godesberg, 24.05.1950. 
tiers. ${ }^{14}$ Ensuite, un «haut collège arbitral» (aussi dénommé «collège des médiateurs») composé de trois membres ${ }^{15}$ aurait été compétent pour trancher les litiges d'ordre économique (recours sur l'opportunité des décisions). ${ }^{16}$ Enfin, on aurait confié à la Cour Internationale de Justice de La Haye le soin de juger le contentieux d'ordre juridique (recours contre les décisions considérées contraires au traité et contre les excès de pouvoirs). ${ }^{17}$ Présenté sans grand détail au comité interministériel français du 12 juin $1950,{ }^{18}$ ce triple mécanisme fut simplifié pendant la semaine précédant l'ouverture des négociations à Six. L'intervention de la Cour de La Haye fut en effet définitivement écartée si bien que, le 21 juin 1950, Monnet n'évoqua que deux procédures d'appel lorsqu'il dévoila aux délégations réunies à Paris les futures institutions du pool. Ces procédures, détaillées aux articles 7 et 8 du projet de traité préparé par les experts français et remis aux cinq autres délégations le 24 juin, méritent notre attention. C'est, en effet, avec ce document dans leurs valises que les négociateurs retournèrent dans leur capitale respective pendant la première interruption de la conférence. Décortiqué par les conseillers juridiques et les responsables politiques, ce premier projet de traité servit ensuite de base de travail aux négociateurs lorsqu'ils se retrouvèrent à Paris à partir du 3 juillet 1950. Voici ce que prévoyaient les deux articles:

1) La Haute Autorité disposerait de la prérogative de recevoir les recours en première instance. Ainsi, un État dont les intérêts seraient mis en cause par une décision ou une recommandation de la Haute Autorité pourrait demander à cette dernière de réexaminer sa position. ${ }^{19}$ Le même droit serait donné aux entreprises mais uniquement pour les décisions ou les recommandations les visant individuellement. La Haute Autorité confirmerait sa décision à la majorité des deux tiers.

2) Un État ou, de manière plus restrictive, une entreprise pourrait ensuite saisir une «Cour d'arbitrage $a d$ hoc». ${ }^{20}$ Les recours ne seraient recevables que dans trois situations spécifiques: lorsqu'un acte du collège supranational viole le traité, lorsqu'il menace de compromettre la politique de plein emploi d'un État ou lorsqu'il met en péril l'équilibre de la balance commerciale d'un pays membre. La

14. FJM, AMG 2/4/4a, Note concernant la Haute Autorité, sans date [s.d.], [mai/juin 1950]; FJM, AMG 2/4/7, Uri à Monnet. Schéma de traité, 07.06.1950; FJM, AMG 2/4/16, Reuter. Observations sur le schéma de traité (article 6), 14.06.1950.

15. Ces trois membres seraient nommés respectivement par le secrétaire général de l'ONU, le président de la Cour Internationale de Justice et le directeur de l'OIT.

16. FJM, AMG 2/4/3, Reuter à Monnet. Projet d'un schéma de traité, 16.05.1950; FJM, AMG 2/4/4a, Note concernant la Haute Autorité, s.d. [mai/juin 1950]; FJM,AMG 2/4/8, Uri. Schéma de traité. Recours contre les décisions de la Haute Autorité, 12.06.1950; Reuter. Observations sur le schéma de traité, 14.06.1950, op.cit.

17. Projet d'un schéma de traité,16.05.1950, op.cit.; Note concernant la Haute Autorité, [mai/juin 1950], op.cit.; Schéma de traité ..., 12.06.1950, op.cit.

18. FJM, AMG 2/2/2, Note pour le Comité interministériel, 12.06.1950.

19. Dans la version du 19 juin, Monnet semble pourtant avoir écarté cette possibilité. (FJM, AMG 2/ $4 / 20)$.

20. Cette cour serait composée de 5 juges: 2 désignés respectivement par la CIJ et le BIT et 3 originaires d'États non impliqués dans le litige, mais membres de la Communauté. 
cour d'arbitrage ad hoc prononcerait une sentence obligatoire pour les parties lorsqu'il s'agit d'un différend portant sur la légalité d'une décision (en cas de violation du traité); dans les autres cas, elle ne jouerait qu'un rôle de médiateur et adresserait à la Haute Autorité une recommandation comportant obligation dans les buts assignés, mais laissant au collège le choix des moyens à adopter pour atteindre ces objectifs. Les recours n'auraient, en principe, pas d'effet suspensif. ${ }^{21}$ Comme son nom l'indique, cette instance d'appel ne formerait pas un organe permanent, du moins pendant une période transitoire. ${ }^{22}$ Convoqués selon les besoins, les juges n'auraient dès lors pas à abandonner leurs activités professionnelles régulières. ${ }^{23}$

Deux constatations s'imposent d'emblée de l'analyse des premiers documents produits par les experts français et de divers témoignages postérieurs. D'abord, une certaine indécision voire un désaccord régnait parmi les Français sur la question des recours. À ce stade, la nature, la composition et le fonctionnement du futur pilier judiciaire de la communauté flottaient encore dans les limbes de leur pensée créatrice. On peut prendre pour preuve de cette hésitation, l'utilisation du conditionnel à l'article 8 , alors que toutes les autres dispositions relatives aux institutions furent rédigées au futur simple. Ensuite, il est clair que si, fidèle aux principes définis dans la déclaration Schuman, l'équipe de la rue Martignac avait bel et bien l'intention d'organiser des voies de recours, elle entendait réduire sensiblement leur importance et leur portée afin d'éviter que les dispositifs d'appel ne portent préjudice à la Haute Autorité. Ainsi, la non-permanence du tribunal arbitral, son simple rôle de médiateur pour les différends non juridiques, sa dénomination même, les délais assez brefs envisagés pour l'introduction d'un recours, le caractère non suspensif des appels constituent autant d'éléments visant à contenir les effets d'un organe perçu comme un élément susceptible de paralyser ou de compromettre le travail de la Haute Autorité.

Dans son souci d'assurer l'indépendance et l'autorité du collège supranational, Monnet a hésité à développer des mécanismes de recours extensifs. Ainsi que le confirma par la suite Maurice Lagrange, l'inspirateur du Plan Schuman craignait l'instauration d'une sorte de "gouvernement des juges». ${ }^{24}$ Il n'entrevoyait certainement pas encore, à ce stade, que l'organe judiciaire pourrait relever du même principe de supranationalité qu'il cherchait à inculquer à la Haute Autorité. Ses craintes ont sans doute été nourries par les analyses des conseillers américains qui, à Paris, à Bonn et à Washington, réfléchissaient eux aussi aux aspects institutionnels du Plan Schuman et en particulier à la question des recours. Le

21. Archives Nationales, Luxembourg/Affaires étrangères (ANL/AE), dos.11347, Document de travail français, 24.06.1950 (articles 7 et 8). Voir aussi Politisches Archiv des Auswärtigen Amts (PAAA), Abt.2, SFSP, dos.62, Kurzprotokoll über Aussprache zwischen H. Monnet und der deutschen Delegation am 22. Juni 1950.

22. PAAA/Abt.2, SFSP, dos.62, Protokoll über die Zusammenkunft der deutschen Delegation mit H. Monnet in Houjarray ..., 02.07.1950.

23. Statische Archieven van het Ministerie van Buitenlandse Zaken (AMBZ) (La Haye), I, 996.1 EG$\mathrm{KS}$, dos.40 Gecombineerde vergadering van de Commissies voor Buitenlandse Zaken en voor de Handelspolitiek op 7 Juli 1950 ...; ANL/AE, dos.11385, Les institutions de la Communauté.

24. Interview de M. Lagrange, op.cit. 
secrétaire d'État Dean Acheson avait certes donné pour consigne qu'aucun schéma institutionnel ne soit suggéré, même de manière officieuse, aux négociateurs européens. Les études des Américains ne restèrent toutefois pas cloîtrées dans les tiroirs des ambassades. ${ }^{25}$ Monnet qui, on le sait, entretenait des liens privilégiés avec les Américains, a été influencé par leurs réflexions sur la gestion des recours sans toutefois adopter la totalité de leurs idées. Plusieurs exemples nous permettront, dans les paragraphes suivants, de prouver cette affirmation.

Les appréhensions de Monnet étaient en revanche loin d'être partagées par les responsables politiques et les experts belges, néerlandais et luxembourgeois. Bien au contraire. Si le commissaire au Plan redoutait qu'un «gouvernement des juges» atténuât le caractère inédit de son initiative, les trois petits pays s'inquiétèrent avant tout de la puissance exorbitante de la nouvelle autorité et cherchèrent à institutionnaliser des garanties efficaces contre ses décisions. Leur inquiétude s'exprima dès leur première rencontre avec Monnet et Schuman, le 24 mai 1950 à Paris. Pressés de questions sur le contrôle de la Haute Autorité, les deux Français laissèrent vaguement entendre que la Cour de La Haye pourrait jouer un rôle en la matière, mais ils restèrent évasifs, remettant aux négociations officielles toute discussion sur la question. ${ }^{26}$

Loin d'apaiser l'anxiété de leurs interlocuteurs, cette attitude incita les trois petits pays à réfléchir aux mécanismes de recours dans une approche globale du problème de la responsabilité de la Haute Autorité. La rencontre du 7 juin entre les fonctionnaires belges, néerlandais et luxembourgeois ne déboucha sur aucune solution tangible quant aux recours, mais l'idée fut émise de confier à des commissaires affectés par les gouvernements auprès de la Haute Autorité la tâche d'introduire les appels auprès d'un organe juridictionnel. ${ }^{27}$ Le Belge Jean-Charles Snoy et d'Oppuers préconisa aussi la création d'un «organisme de conciliation et d'arbitrage» (ou une «Cour du contentieux»), inspirant confiance et capable d'intervenir rapidement si une décision de la Haute Autorité menaçait les «intérêts vitaux» d'un État ou était «incompatible avec sa souveraineté». ${ }^{28}$ Parce qu'elle ne soumettait les décisions de la Haute Autorité qu'au contrôle d'une instance juridique et non à celui d'un organe politique, cette solution ne satisfit pas les Néerlandais. ${ }^{29}$

25. National Archives and Records Administration (NARA), RG 466, McCloy's Papers, Classified General Records 1950, dos.13, Acheson à Paris et Francfort, 08.07.1950.

26. ANL/AE, dos.11346, Hommel. Compte-rendu de la séance d'information du 24 mai 1950 [...], 25.05.1950.

27. AMBZ, I, 996.1 EGKS, dos.38, Mémorandum de van Voorst tot Voorst à van der Brink, 10.06.1950; Ibid., Verslag van de bespreking te Brussel op Woensdag 7 Juni inzake Schuman-Plan, 10.06.1950; ANL/AE, dos.11346, Calmes et Conrot. Réunion entre les fonctionnaires [...], 08.06.1950.

28. Archives du Ministère des Affaires étrangères belge (AMAE/B), Dos.gén. CECA 5216, Projet de traité réalisant le Plan Schuman. Principales dispositions à prévoir, s.d.; Réunion du Plan Schuman - 7 juin 1950 (délégations belgo-luxembourgeoise et néerlandaise).

29. Verslag van de bespreking te Brussel ..., 10.06.1950, op.cit. 
Les premiers jours des négociations à six et le document de travail français du 24 juin ne firent que conforter le désir des trois petits pays de mettre en place un dispositif protégeant davantage les intérêts des États contre une Haute Autorité jugée toute puissante. À Luxembourg, on suggéra que la Cour d'arbitrage fût composée des ministres des Affaires économiques qui choisiraient un magistrat de la Cour Internationale de Justice comme président. On exigea aussi l'élargissement du droit de recours (voir infra). ${ }^{30}$ Le gouvernement de La Haye imagina un scénario institutionnel analogue. Il prévoyait l'instauration d'un Comité ministériel, composé des ministres des Affaires économiques, agissant comme instance de premier appel. Ce comité confirmerait par un verdict arrêté à la majorité des deux tiers ou des trois quarts une décision contestée de la Haute Autorité. Par ailleurs, ils réclamèrent la création d'une «Cour d'arbitrage» ou l'utilisation d'une «instance internationale appropriée» ${ }^{31}$ pour juger, de manière définitive, les appels en seconde instance. ${ }^{32}$

Le 3 juillet, quelques heures après en avoir discuté avec ses homologues belge (Max Suetens) et luxembourgeois (Albert Wehrer), le chef de la délégation néerlandaise, Dirk Spierenburg expliqua donc aux délégations à nouveau réunies à Paris que son gouvernement acceptait le principe d'une Haute Autorité supranationale, mais jugeait indispensable d'associer les États au contrôle de cet organe par le biais d'un Comité des ministres, chargé des recours en première instance. ${ }^{33}$ Cela dit, il importe de comprendre que les Néerlandais ne tenaient pas impérativement à transformer le Comité des ministres en une cour d'appel. Ce qui les intéressait avant tout, c'était de ramener dans la structure institutionnelle du Plan Schuman, d'une manière ou d'une autre, les gouvernements exclus par les Français. Les idées présentées par Spierenburg le 3 juillet (et à nouveau le 5 juillet) ne constituaient donc pas «une contre-proposition mais une suggestion faite à titre indicatif». .34

Appuyée par les Luxembourgeois et les Belges, la proposition néerlandaise, on le sait, mobilisa les débats initiaux de la conférence de Paris. Celle-ci s'organisa en cinq groupes de travail pour étudier les problèmes concrets liés à la mise en application du plan. Le premier de ces groupes, composé des chefs de délégation, examina les questions institutionnelles. Il fallut environ dix jours de réflexion créatrice pour démêler l'écheveau institutionnel et trouver une manière de réconcilier les exigences néerlandaises à la nécessité de préserver le caractère supranational de la Haute Autorité. Au terme d'une semaine de discussions

30. ANL/AE, dos.11347, Plan Schuman. Considérations du Département des Affaires Économiques [...]; Plan Schuman. Entrevue du 3 juillet [...].

31. FJM, AMG 3/3/17, Conversations sur le Plan Schuman. Séance restreinte. Lundi après-midi 3 juillet 1950 (PS/CR5).

32. Entrevue du 3 juillet ..., op.cit.; AMBZ, I, 996.1 EGKS, dos.40, Kort verslag van de bespreking inzake het Plan Schuman met de Belgische en Luxemburgse delegaties [...].

33. FJM, AMG 3/3/17, Conversations sur le Plan Schuman (PS/CR5).

34. FJM, AMG 4/1/1, GT 1, Conversations sur le Plan Schuman. Réunion du Comité des Chefs de délégation [...], mercredi matin 5 juillet 1950. 
officieuses avec les différentes délégations, Monnet finit par reconnaitre que l'architecture institutionnelle esquissée dans le document de travail du 24 juin comportait une lacune grave et, avec pragmatisme, il accepta l'ajout d'un organe permettant l'expression des intérêts nationaux. ${ }^{35} \mathrm{Il}$ rejeta cependant promptement la suggestion néerlandaise initiale de confier au comité ministériel la tâche de procéder à une seconde lecture des décisions litigieuses du collège supranational et de les confirmer à une majorité des deux tiers voire des trois quarts. Les Allemands n'en voulaient pas non plus. ${ }^{36}$ Cette formule aurait en effet mis le Conseil des ministres en position d'entraver très sérieusement l'activité de la Haute Autorité; elle fut donc rapidement abandonnée et les négociateurs établirent d'autres liens organiques entre le collège supranational et l'instance défendant les intérêts nationaux, liens qu'il ne nous appartient pas de développer dans cet article. Signalons enfin que John McCloy et, par la suite, Dean Acheson lui-même rejetèrent aussi l'idée de confier à un conseil ministériel la deuxième lecture des décisions contestées de la Haute Autorité car cette formule mettait en péril le principe - qu'ils savaient crucial - de la supranationalité. ${ }^{37}$ Même si l'on peut raisonnablement penser que Monnet et ses collaborateurs arrivèrent personnellement à la même conclusion, la condamnation américaine les a très certainement confortés dans leur refus de considérer l'option néerlandaise.

À partir du moment où, vers la mi-juillet 1950, l'ajout d'un Conseil spécial de ministres fut décidé et son rôle clarifié, les six délégations purent entamer des discussions sérieuses sur les mécanismes de recours. Les questions étant complexes, les chefs de délégation transmirent le «dossier institutionnel» aux spécialistes du droit. ${ }^{38}$ Monnet rappela Reuter à Paris et le plaça à la tête d'un comité des juristes, composé des experts juridiques de chaque délégation. ${ }^{39}$ Sans perdre de temps, ce comité analysa, article par article, le document de travail du 24 juin afin de dégager les points de convergence entre les délégations. Le 26 juillet, après huit séances, ${ }^{40}$ il présenta aux chefs de délégation son rapport ainsi qu'une série de dispositions sur lesquelles un accord provisoire avait été conclu. ${ }^{41} \mathrm{Ce}$

35. PAAA/Abt.2, 221-40, dos.745, Hallstein à Blankenhorn, 12.07.1950.

36. PAAA/Abt.2, SFSP, dos.17, Ophüls. Arbeitsaufzeichung. Betr.: Unmittelbare Entscheindungsgewalt der Regierung, 10.07.1950

37. NARA, RG 466, McCloy's Papers, Classified Gen. Rec.1950, dos.13, Tél.175, McCloy au Dép. d'État, 07.07.1950; Foreign Relations of the United States, 1950, vol.III, Bureau of Public Affairs, Washington, 1977, pp. 741-742, Acheson à McCloy, 25.07.1950.

38. FJM, AMG 4/1/3, GT 1, Projet de compte rendu (PS/G1/CR3).

39. Pour l'Allemagne: Blankenhorn (conseiller personnel d'Adenauer), Ophüls (ministère de la Justice) et H. Mosler; pour la Belgique: Muûls (jurisconsulte au MAE) et Cassiers; pour la France: Clappier, Reuter, Gros, et Hubert (conseillers juridiques au MAE); pour l'Italie: Santoro (conseiller d'État), Venturini (conseiller de Légation), De Rossi (du MAE) et Balladore-Pallieri (du ministère du Trésor); pour le Luxembourg: Calmes et enfin pour les Pays-Bas: Kohnstamm, Francois, Blaisse et Riphagen. Le comité fut présidé par Reuter, Hubert et Gros.

40. Pour un bref compte rendu officieux de ces réunions, voir PAAA/Abt.2, SFSP, dos.18 et 19.

41. FJM, AMG 4/1/5, GT 1, Réunion du Comité des Chefs de délégation sur les questions institutionnelles, mercredi après-midi 26 juillet 1950 (PS/G1/CR5); FJM, AMG 4/1/5bis, GT 1, Note sur les résultats des travaux du Comité des juristes à la date du 25 juillet 1950. 
rapport et les discussions qu'il suscita au niveau des chefs de délégation permirent alors à Reuter de préparer, en collaboration étroite avec Monnet, un «Mémorandum sur les institutions» ${ }^{42}$ qui, après révision suite aux commentaires des autres délégations, fut adopté d'un commun accord et joint au rapport général sur l'état des travaux, daté du 10 août $1950 .{ }^{43}$

L'organisation des recours proposée dans ce nouveau document de travail comportait des modifications essentielles par rapport à ce que les Français avaient initialement imaginé. Premier changement important: il n'était plus question de faire de la Haute Autorité l'instance de premier appel, chargée de procéder à une deuxième lecture de ses propres décisions. Aucun document d'archive ne fournit d'explications définitives quant aux raisons de cet abandon, mais on peut évoquer différents facteurs ayant probablement contribué à cette décision. Il faut d'abord souligner que le mémorandum du 10 août écartait la possibilité d'introduire un recours fondé sur l'opportunité d'une décision prise par le collège supranational (voir infra), rendant dès lors inutile un mécanisme d'appel obligeant la Haute Autorité à reconsidérer ses décisions pour des raisons politico-économiques. On peut également supposer que les délégations du Benelux, déjà alarmées par la toute puissance de la Haute Autorité, se sont opposées à cette procédure. À cela s'ajoutent aussi les sérieuses réserves exprimées par les Américains qui considéraient que le prestige de la Haute Autorité serait mis à mal si cette dernière était régulièrement invitée à renverser ses décisions, chaque retrait d'un acte déjà promulgué affaiblissant sa crédibilité. ${ }^{44}$ On peut imaginer l'effet d'un tel raisonnement sur la pensée de Monnet.

Enfin, et ceci est important, il était devenu clair à ce stade des travaux que la Haute Autorité ne constituerait pas la seule institution du pool mais qu'elle fonctionnerait dans le cadre d'une communauté, en collaboration avec d'autres organes (Conseil et Assemblée). Dans un tel contexte, la nécessité de doter cette nouvelle entité internationale d'un organe juridictionnel unique et propre s'imposa de plus en plus clairement aux juristes. Ils écartèrent donc l'idée de multiplier les procédures de recours et renoncèrent à utiliser comme instance d'appel la Haute Autorité ou les tribunaux internationaux déjà en place. Un seul et même organe serait chargé de trancher tous les litiges introduits tant contre les décisions de la Haute Autorité que - nouveauté du mémorandum - contre celles de l'Assemblée commune et du Conseil spécial de ministres. ${ }^{45}$ Ces principes établis, les Six

42. ANL/AE, dos.11349, Premier avant-projet. Mémorandum sur les institutions de la proposition du 9 mai, 01.08.1950. Cf. aussi la première rédaction de ce document: Centre d'Accueil et de Recherche des Archives Nationales(CARAN), Commissariat Général du Plan, Série 81 AJ, dos.159, Mémorandum sur les institutions de la proposition du 9 mai (Plan Schuman), 28.07.1950.

43. ANL/AE, dos.11384, Mémorandum sur les institutions (annexe I du Rapport sur les travaux poursuivis à Paris [...] du 20 juin au 10 août 1950).

44. McCloy au Dép. d'État, 07.07.1950, op.cit.; NARA, RG 84, Paris Embassy, Classified Gen. Rec.1950, dos.71, Working Group on Schuman Proposal. Check list on first Draft of Working Document, 13.07.1950.

45. FJM, AMG 4/1/5 bis, GT 1, Note sur les résultats [...]; ANL/AE, dos.11384, Plan Schuman. Rapport d'ensemble sur l'état des négociations au $1^{\mathrm{er}}$ août 1950. 
arrêtèrent définitivement le nom de l'instance de recours: à partir de la fin juillet, ils parlèrent exclusivement de la «Cour de Justice». ${ }^{46}$

L'abandon définitif du caractère ad hoc de l'instance arbitrale constitue le second changement clé consacré dans le mémorandum du 10 août. Sous l'influence conjuguée des Américains et du chef de la délégation allemande, les Français acceptèrent l'instauration d'une Cour permanente. ${ }^{47}$ Walter Hallstein, un professeur de droit qui en 1948 avait passé une année à l'université Georgetown (Washington, DC), fut un des premiers à entrevoir les bénéfices d'une cour de justice permanente. «Gardienne de l'objectivité de la Haute Autorité», elle remplirait une «fonction éducatrice considérable», expliqua-t-il à Monnet lors de leur rencontre à Houjarray, le 2 juillet, à la veille de la reprise des négociations de Paris. ${ }^{48}$ Il réussit à convaincre Bernard Clappier, semble-t-il. Mais Monnet resta réticent et exprima ses craintes de voir un tel tribunal prendre rapidement le dessus sur la Haute Autorité. Il reconnut toutefois la validité des idées de son interlocuteur.

Par la suite, différents facteurs contribuèrent progressivement à atténuer ses craintes et à infléchir sa position. Il dut bien sûr tenir compte des exigences de ses partenaires à la table des négociations car, outre les Allemands, les autres délégations - la belge en particulier - insistèrent sur la permanence de l'organe juridictionnel. L'influence américaine est également indéniable: tant Robert Bowie que George Ball, David Bruce ou John McCloy appuyaient l'idée d'une Cour permanente, compétente exclusivement pour le contentieux juridique. Se référant à l'exemple de la Cour Suprême aux États-Unis, Ball mit certes Monnet en garde contre le risque de voir les juges permanents réinterpréter, au fil des années, le traité et les intentions de ses auteurs, mais il conclut que, si le traité était rédigé sans ambiguïté, on éviterait ce dérapage et les bénéfices issus d'une interprétation consistante du droit surpasseraient largement ce risque potentiel ${ }^{49} \mathrm{Ce}$ point de vue était partagé par Hallstein qui expliqua d'ailleurs à Monnet combien il valorisait l'opinion américaine sur la question des recours et lui rappela la satisfaction suscitée outre-Atlantique par cette composante institutionnelle, perçue comme un premier pas vers la séparation des pouvoirs au sein d'un État fédéral en devenir.

La position de Monnet en faveur d'une cour permanente évolua aussi parallèlement à sa conception du droit de recours. À partir du début juillet, il considéra en effet que les recours pour motifs économico-politiques ne devraient pas être autorisés (voir infra). On peut raisonnablement penser que la limitation des compétences de la Cour aux seuls litiges juridiques aida Monnet à accepter plus facilement sa permanence. Enfin, il importe de mentionner l'influence que Reuter

46. PAAA/Abt.2, SFSP, dos.18, Kurzprotokoll des Juristischen Ausschusses über die Sitzung vom 24.7.1950 (nachmittags).

47. Plan Schuman. Rapport d'ensemble [...]; PAAA/Abt.2, SFSP, dos.53, Notizen. Hallstein à von Brentano, 08.08.1950.

48. PAAA/Abt.2, SFSP, dos.62, Protokoll über die Zusammenkunft der deutschen Delegation mit H. Monnet in Houjarray, 02.07.1950.

49. FJM, AMG 4/6/6, B[all]. Memorandum of suggestions regarding article 8 of proposed working paper, 04.07.1950. 
exerça à ce stade des négociations sur Monnet et sur l'édification de la structure institutionnelle. Le mémorandum du 10 août fut en effet, pour l'essentiel, l'œuvre du professeur de droit d'Aix-en-Provence. Très étroitement associé à l'élaboration de la déclaration du 9 mai, il était intimement persuadé de l'importance de la supranationalité. Sa formation académique l'incita à envisager le carré institutionnel en émergence dans une perspective théorique. Si, comme d'autres, il vit dans la nouvelle structure institutionnelle les prémices d'un système fédéral, il considérait que c'était la Cour - et non le Conseil - qui, avec la Haute Autorité, s'apparentait le plus à une institution fédérale. «La Haute Autorité et la Cour de Justice», souligna-t-il,

«sont composées toutes les deux de personnalités absolument indépendantes, qui ne doivent garder aucun lien avec les États. À cet égard, elles constituaient une réalisation très avancée et, pour ainsi dire, parfaite de la communauté supranationale». ${ }^{50}$

Le Conseil de ministres et l'Assemblée commune ne constituent par contre à ses yeux qu'une réalisation «moins avancée». ${ }^{51}$ Cette analyse aida probablement Monnet à considérer la Cour sous un nouvel angle, à la voir non pas comme une menace pour la Haute Autorité, mais comme une composante institutionnelle essentielle, capable de contribuer au développement de la nouvelle communauté. Il resta néanmoins très vigilant à son égard.

Tous les juristes au sein de la délégation française ne partageaient cependant pas cette vision. À l'automne 1950, en profitant de la rédaction d'un nouveau document de travail destiné à fixer les acquis des négociations, certains tentèrent de revenir sur l'accord dégagé en août. Le 8 novembre 1950, les Français soumirent en effet un projet de traité dont l'article 25 établissait qu'initialement du moins, les juges n'exerceraient pas leurs fonctions à temps plein et qu'il reviendrait au Conseil de ministres de modifier cette situation s'il l'estimait nécessaire. Cette disposition fut inspirée par le conseiller juridique Lucien Hubert pour qui l'instauration, dans une phase initiale, d'une «simple commission arbitrale» permettrait de réduire les dépenses et de se donner du temps en faisant «un essai plus modeste des institutions avant d'engager l'avenir, notamment par le choix des personnes». ${ }^{52} \mathrm{La}$ proposition française fut naturellement très mal reçue ${ }^{53}$ par les cinq délégations qui trouvèrent cependant une oreille favorable auprès d'autres Français, en particulier Hirsch et Lagrange. Soucieux d'assurer le prestige de la Cour, ce dernier se prononça en faveur de juges exerçant leurs fonctions communautaires à plein temps. ${ }^{54}$ La tentative de postposer la permanence de la Cour fut alors

50. Mémorandum du 10 août 1950.

51. Cette expression apparaît dans le projet de mémorandum du 28 juillet (CARAN, Commissariat Général du Plan, Série 81 AJ, dos.159), mais n'est reprise ni dans la version du $1^{\text {er }}$ août ni dans la version finale du 10. L'analyse de Reuter fut contestée par les Allemands qui voyaient dans l'Assemblée un élément clé de la future fédération européenne.

52. FJM, AMG 6/5/15, Hubert. Note confidentielle sur la Cour de Justice, 27.09.1950.

53. AMAE/B, Dos.gén. CECA 5216, F. Muûls. Note concernant le projet de traité relatif au charbon et à l'acier, 16.11.1950.

54. AMAE/B, Dos.gén. CECA 5216, Note à Monsieur le Ministre Muûls, Paris, 21.11.1950. 
définitivement écartée et le projet de traité du 27 novembre 1950 ne reprit plus les dispositions incriminées.

\section{De la composition et des compétences de la Cour de Justice}

Les articles du traité de Paris consacrés à la Cour de Justice (art.31 à 45) furent, selon le témoignage de Lagrange, rédigés en une vingtaine de jours et, à la différence d'autres questions institutionnelles, ne nécessitèrent pas d'arbitrage ministériel. Ces propos sont exacts. Il n'en reste pas moins que la composition et les compétences de la Cour suscitèrent des débats passionnés que nous voudrions maintenant évoquer.

Le nombre de juges à appointer et le mode de leur désignation soulevèrent des problèmes analogues à ceux liés à la composition de la Haute Autorité mais d'une intensité moins grande. ${ }^{55}$ Le document de travail français du 24 juin 1950 proposait, à titre indicatif, que la Cour d'arbitrage ad hoc comprenne cinq membres: trois désignés par les gouvernements, dans la mesure du possible issus d'États non impliqués dans l'affaire traitée ou étrangers à l'entreprise faisant appel, et deux nommés respectivement par la Cour Internationale de Justice et l'Organisation Internationale du Travail. ${ }^{56}$ Le gouvernement néerlandais souhaitait quant à lui que la Cour compte autant de juges qu'il y a d'États membres, plus deux juges nommés par la Cour Internationale et l'Organisation Internationale. ${ }^{57}$ Les juristes allemands modifièrent leur position au cours des négociations: initialement ils recommandaient la nomination de neuf juges et de neuf suppléants, élus pour six ans par l'Assemblée commune, ${ }^{58}$ en août 1950 , ils optèrent pour une Cour de sept membres nommés par les gouvernements. ${ }^{59}$

Bien que ces combinaisons n'aient pas été préalablement discutées par les chefs de délégation, les Français introduisirent unilatéralement dans le projet de traité du 8 novembre 1950 plusieurs précisions importantes quant à la composition de la Cour: elle comprendrait cinq juges titulaires et deux suppléants, choisis parmi des «personnalités offrant toutes garanties d'indépendance et de compétence» et nommés pour six ans d'un commun accord par les gouvernements. Leur nombre pourrait être augmenté par le Conseil des ministres, statuant à l'unanimité. Tous les trois ans, les juges titulaires désigneraient leur président. L'essentiel de ces dispositions fut repris à l'article 32 du traité de Paris. Une différence de taille existe

55. A. BOERGER-DE SMEDT, Derrière la valse des chiffres. Retour sur les origines de la composition de l'exécutif européen, in: Revue d'histoire de l'intégration européenne, 1(2007), pp.25-48.

56. ANL/AE, dos.11347, Doc. de travail français (Art.8), 24.06.1950.

57. AMBZ, I, 996.1 EGKS, dos.40, Gecombineerde vergadering van de Commissies voor Buitenlandse Zaken en voor de Handelspolitiek.

58. PAAA/Abt.2, SFSP, dos.4, Kraus. Mittagssitzung der Juristen-Kommission vom 28. Juni 1950.

59. PAAA/Abt.2, SFSP, dos.105, Entwurf für Richtlinien für die weiteren Beratungen über institutionelle Fragen, 22.08.1950; dos.102, BMWi. Unterlagen zum Schuman-Plan, 17.08. 1950. 
cependant entre les deux documents: le traité final prévoyait la nomination de sept juges au lieu de cinq et ne mentionnait pas les suppléants. Le nombre de magistrats européens fut augmenté pour satisfaire les exigences du Luxembourg qui, pour la Cour comme pour la Haute Autorité, craignait d'être tenu à l'écart des nouvelles institutions. ${ }^{60}$ Le nombre de sept semblait donc idéal aux fondateurs de la CECA puisque, sans être identique à celui des pays membres, il permettait à chaque gouvernement d'appointer un de leurs ressortissants à la Cour européenne. Le traité de Paris ne précisait toutefois pas que les membres de la Cour devaient obligatoirement ressortir d'un des pays membres de la Communauté alors qu'une telle clause existait pour la Haute Autorité. Ce serait une erreur toutefois de conclure que les négociateurs du traité de Paris envisagèrent d'installer à Luxembourg des juges provenant d'un État non membre de la Communauté. Ainsi que Lagrange l'expliqua par la suite, l'absence de référence à la nationalité des juges constitue une simple omission causée par le fait que les articles relatifs à la Cour, plus anciens, étaient déjà paraphés lorsque, en dernière minute, les ministres ont arrêté les dispositions relatives à la Haute Autorité. Les raccords de texte n'ont pas pu être effectués. ${ }^{61}$

Ainsi, si théoriquement le traité permettait la désignation de juges choisis en dehors du pool charbon-acier, un tel scénario restait une pure vue de l'esprit car il était bel et bien acquis, dès avant la signature du traité, que la Cour comprendrait au moins un ressortissant de chaque pays membre et que le septième siège de juge serait attribué à un représentant des syndicats ouvriers. On peut en effet difficilement imaginer qu'un gouvernement se serait volontairement privé des assurances offertes par la présence d'un de ses nationaux à la Cour. La composition de la première Cour refléta cet accord tacite: chaque État, grand ou petit, envoya un juge à Luxembourg et le septième juge fut un syndicaliste; les deux avocats généraux furent français et allemand (Lagrange et Karl Roemer) et, pour satisfaire les revendications de Rome, le juge italien (Massimo Pilotti) présida la Cour. En 2001, les Quinze enchâssèrent finalement dans le traité de Nice la formule d'«un juge par État membre» (art.221), officialisant ainsi une pratique tacitement établie dès les origines de l'Union.

D'autres principes introduits par les Français dans le projet de traité du 8 novembre suscitèrent également des discussions intéressantes même si, en fin de compte, ils ne furent pas ou peu modifiés. Ainsi Muûls s'opposa à la désignation des juges par les gouvernements et demanda - en vain - que la durée de leur mandat fusse portée à 9 ans. Selon lui, les conditions de nomination proposées par les Français plaçaient les juges à la merci de la bonne volonté des ministres, ce qui semblait incompatible avec le principe d'indépendance, affirmé à l'article 32 du traité. $^{62}$ Ceci lui paraissait d'autant plus alarmant que les juges n'étaient pas

60. ANL/AE, dos.11385, Les institutions de la Communauté; dos.11352, Projet de traité. Institutions et Dispositions générales. Art.1 à 31 bis et 52 à 70, 30.11.1950.

61. Archives du Ministère des Affaires étrangères/France (AMAE/F), DE-CE, dos.503, Compte-rendu de l'assemblée générale du Conseil d'État tenue le 2 mai 1951.

62. F. Muûls. Note concernant le projet de traité ..., 16.11.1950, op.cit. 
nommés à vie et que le renouvellement de leurs mandats était, lui aussi, laissé à la discrétion des gouvernements. Ce point de vue était valide et d'ailleurs partagé par certains Allemands. ${ }^{63}$ Appuyée par les autres délégations, la procédure proposée par la France a pourtant prévalu. Reuter la justifiait par deux arguments: d'une part, elle réservait aux gouvernements «le rôle qui leur revenait» et, d'autre part, elle permettait des «conversations d'ensemble» sur les désignations à la Cour et à la Haute Autorité, conversations «où les satisfactions [pourraient] être négociées en faveur de tous les États membres». ${ }^{64}$

Reuter ne précisa pas quel était, selon lui, ce «rôle» qui revenait aux gouvernements. S'agissait-il de garder la main haute sur la Cour en contrôlant qui y siègerait et en s'assurant qu'un juge devenu indésirable puisse être écarté avec son premier mandat? Probablement. Le mode de désignation des juges et le caractère provisoire de leur emploi offrent des garanties rassurantes pour les gouvernements. La procédure choisie répondait en tout cas davantage à des préoccupations politiques qu'à des préférences juridiques. La nomination à vie des juges n'était toutefois pas considérée par tous les juristes comme une indispensable garantie d'indépendance. Certains firent valoir que les Allemands et les Italiens, par exemple, n'avaient pas retenu ce principe pour leur cour constitutionnelle respective. Quoiqu'il en soit, l'indépendance des magistrats européens fut donc simplement stipulée dans le traité sans être vraiment garantie. Certains ont par la suite, expliqué que c'est en fait le secret des délibérations qui assure l'autonomie des juges. ${ }^{65}$

Les négociateurs ont en effet refusé d'adopter le système de l'opinion dissidente, peu connu sur le continent, mais appliqué dans certaines juridictions anglo-saxonnes et à la Cour Internationale de La Haye. Les verdicts de la Cour CECA se présentent donc comme des décisions collégiales et le secret du délibéré assure que l'opinion personnelle d'un juge ne soit jamais révélée, l'abritant ainsi de toute pression politique. La validité de cette analyse est incontestable. Il est à noter toutefois que, selon le témoignage de Lagrange, la règle de l'opinion dissidente ne fut discutée qu'en toute fin de négociations et écartée malgré l'insistance des Hollandais. Ce fut d'ailleurs dans ce contexte que le juriste français introduisit les avocats généraux, une institution directement inspirée du Conseil d'État français. ${ }^{66}$ Elle fut présentée «comme une sorte de contre-partie à l'interdiction du droit pour les juges de publier éventuellement leur opinion dissidente». ${ }^{67}$

63. PAAA/Abt.2, SFSP, dos.5, Protokoll über die Sitzung des Koordinierungsausschusses für den Schuman-Plan, 7. Dezember 50.

64. FJM, AMG 12/2/2, Reuter. Observations sur les problèmes politiques réservés aux entretiens directs entre gouvernements, 06.02.1951.

65. N. CATALANO, Manuel de droit des Communautés européennes, Dalloz et Sirey, Paris, 1965, p.65.

66. M. LAGRANGE, La Cour de Justice ..., op.cit., p.129; Interview de M. Lagrange, op.cit. Aucun document d'archive ne permet de confirmer ou d'infirmer ce témoignage.

67. Discours de M. Lagrange à l'audience solennelle de la Cour, 08.10.1964 (http:// www.ena.lu?lang $=1 \& d o c=8791$ ). 
Venons-en maintenant aux compétences de la Cour. Dès le début des négociations, la question de la recevabilité des recours mobilisa l'attention des négociateurs. Initialement, nous l'avons dit, les Français prévoyaient qu'un appel pourrait être introduit si une décision ou une recommandation de la Haute Autorité (1) violait les dispositions du traité, (2) mettait en péril l'équilibre de la balance commerciale d'un État ou (3) menaçait la politique de plein emploi d'un pays. Dans le premier cas, ce serait donc la légalité de la décision qui serait contestée, dans les deux autres, son opportunité. ${ }^{68}$ Au début du mois de juillet, Monnet réduisit de manière significative ces dispositions en écartant la possibilité de recours fondés sur l'opportunité. Il s'en expliqua confidentiellement à Hallstein le 12 juillet, argumentant que si la Cour était habilitée à se prononcer sur le bien-fondé économico-politique des décisions de la Haute Autorité, elle se substituerait vite à celle-ci. ${ }^{69}$

Cette analyse devint rapidement la position officielle de la délégation française. Pour justifier cette réduction du droit d'appel, Monnet fit valoir que la notion de recours d'opportunité, initialement introduite pour faciliter l'adhésion des Britanniques au Plan Schuman, était rendue caduque par l'ajout d'un Conseil de ministres veillant aux intérêts vitaux des États. ${ }^{70}$ Il est clair que Monnet fut aussi influencé par les juristes américains. Dans leur analyse respective du document de travail français du 24 juin 1950, Robert Bowie ${ }^{71}$ et George Ball ${ }^{72}$ avaient en effet insisté sur la confusion créée par le fait que le même tribunal d'arbitrage, opérant sur des modes différents, assurait tant le contrôle de la légalitéque celui de l'opportunité de décision de la Haute Autorité. ${ }^{73}$ Selon l'analyse américaine, l'autorité et le standing de la Haute Autorité seraient irrévocablement minés si un collège de médiateurs était habilité à juger le bien-fondé de ses décisions et, le cas échéant, à se prononcer contre leur maintien. Cela provoquerait rapidement l'émergence d'une autorité supérieure au collège supranational. Ils proposèrent qu'un État inquiet des troubles graves qu'une décision de la Haute Autorité engendrerait dans son économie soumette plutôt sa plainte à l'Assemblée commune. Cette alternative ne fut pas considérée par les Français, mais le doute était semé, en particulier dans l'esprit de Monnet, quant à sagesse du système initialement imaginé.

À des années lumières de ce raisonnement, les autres délégations dénoncèrent par contre le caractère restrictif des recours prévus par les Français, et cela avant

68. FJM, AMG 3/2/1, Conversations sur le Plan Schuman. Compte-rendu de la seconde séance tenue le 21 juin 1950; ANL/AE, dos.11347, Document de travail français, 24.06.1950.

69. Hallstein à Blankenhorn, 12.07.1950, op.cit.

70. FJM, AMG 4/1/4, GT 1, Réunion du Comité des Chefs de délégation sur les questions institutionnelles, ... 21 juillet 1950.

71. Professeur de droit constitutionnel à Harvard et spécialiste de la législation antitrust, Robert Bowie était le conseilleur juridique du Haut-commissaire américain en Allemagne, John McCloy.

72. Ami de longue date de Monnet, Ball était conseiller juridique au Commissariat du Plan.

73. CARAN, Série 81 AJ, dos.161, Lettre et mémorandum de Bowie à Monnet, 01.07.1950; Memorandum of suggestions regarding article 8 ..., 04.07.1950, op.cit. John McCloy partageait cette analyse (NARA, RG 466, Tél.175, 07.07.1950.) 
même que Monnet ne les réduise encore. Ainsi, le Luxembourg exigea qu'une procédure d'appel puisse être engagée chaque fois qu'un intérêt vital d'un État serait menacé par une action de la Haute Autorité. ${ }^{74}$ Non défini, ce concept de «mise en cause d'un intérêt vital» était assez vaste pour couvrir toutes les situations mettant en danger l'économie luxembourgeoise. Cette revendication devint vite un des chevaux de bataille de la délégation grand-ducale. Des considérations similaires furent exprimées par les Belges ${ }^{75}$ et les Allemands. ${ }^{76}$

Des discussions entre les chefs de délégation ${ }^{77}$ et au sein du comité des juristes ${ }^{78}$ permirent néanmoins de dégager quelques points d'accord que Reuter consigna dans le mémorandum du 10 août 1950. Le défi des juristes fut de donner à la Cour les moyens de contribuer, par sa jurisprudence, au développement de la Communauté tout en évitant que «ses arrêts soient l'instrument d'un véritable transfert de pouvoirs de la Haute Autorité vers la Cour de Justice». Une «rigoureuse séparation des pouvoirs» devait produire ce résultat. Il fut donc établi que la Cour serait habilitée à annuler les décisions et recommandations de la Haute Autorité non conformes «aux termes et à l'esprit du traité» et à constater, par un arrêt déclaratoire, une carence si l'organe supranational refusait de prendre les décisions que le traité lui imposait de prendre. En toute logique, ce contrôle juridictionnel devait s'appliquer aussi à l'action du Conseil spécial de ministres et de l'Assemblée commune. La Cour ne disposant que d'un pouvoir d'annulation, il ne lui appartiendrait pas d'élaborer de nouvelles dispositions en lieu et place de celles qu'elle infirmerait. Personne ne contesta ces principes.

Beaucoup plus controversée, par contre, fut la possibilité de contester une décision sur base de son inopportunité économico-politique. Sur ce point, la conception française s'imposa: cette possibilité fut en effet exclue car elle «dépouillerait la Haute Autorité de tout pouvoir propre». ${ }^{79}$ Sur l'insistance des Luxembourgeois en particulier, les auteurs du mémorandum acceptèrent toutefois de prévoir un article garantissant l'obligation pour la Haute Autorité de ne pas provoquer des «troubles fondamentaux et persistants» par rapport à l'équilibre de la balance des comptes, au maintien du revenu national et au niveau de l'emploi dans chaque État. Saisie sur base de cet article, la Cour pourrait annuler une décision de la Haute Autorité et une procédure de médiation serait organisée selon des modalités qui restaient à discuter. ${ }^{80}$

La question des compétences de la Cour ne fut guère plus abordée par les négociateurs avant l'entrée en scène de Lagrange et la rédaction du projet de traité

74. ANL/AE, dos.11374, Déclaration de Wehrer à la réunion du 22 juin 1950; dos.11347, Exposé de Monsieur Monnet du 21 juin 1950; Plan Schuman. Considérations du Département des Affaires Économiques; Plan Schuman. Entrevue du 3 juillet à 15 heures.

75. ANL/AE, dos.11372, [F. Muûls]. Recours, 23.06.1950.

76. PAAA/Abt.2, SFSP, dos.61, Ophüls. Rechtsmittel, 28.06.1950; dos.4, Kellermann. Vormittagssitzung der Juristen-Kommission vom 29. Juni 1950.

77. FJM, AMG 4/1/3, GT 1, Projet de compte-rendu (PS/G1/CR3).

78. FJM, AMG 4/1/5 bis, GT 1, Note sur les résultats ....

79. Mémorandum du 10 août 1950, op.cit.

80. Idem. Les Belges et les Allemands rejetèrent l'idée de Reuter d'associer l'Assemblée commune lors du processus de médiation. 
du 8 novembre $1950{ }^{81} \mathrm{Il}$ a été clairement établi - d'abord par lui-même - que, pour rédiger les articles 26 à 29, Lagrange s'inspira très largement du droit administratif français et des pratiques du Conseil d'État dont il était issu. Ce serait ainsi lui qui aurait établi l'analogie entre le rôle du juge européen et celui du juge administratif. Ce point ressort très clairement par exemple dans une interview accordée à Antoine Marès en 1980: Monnet, se souvint Lagrange,

«avait senti que cette affaire touchait au Conseil d'État alors que, jusqu'ici, les négociateurs qui n'avaient pas compris la nature tout à fait nouvelle et révolutionnaire du Plan Schuman, avaient conçu cette Cour de Justice comme une espèce de Cour internationale, genre Cour de La Haye. Moi, issu du Conseil d'État, j'ai tout de suite compris - ce n'était pas difficile - qu'il s'agissait de contrôler la légalité des décisions de la Haute Autorité et de donner à cette Cour, à ces juges, un pouvoir d'annulation sur les décisions contraires au traité, tout en réservant le contrôle de l'opportunité selon la distinction classique». ${ }^{82}$

La contribution de Lagrange fut certes majeure: c'est lui qui aida à définir les compétences de la Cour et à mettre en forme les articles relatifs s'y rapportant. Il introduisit aussi, en fin de négociation, les avocats généraux, propres au système français. De même, les pratiques et la terminologie du Conseil d'État servirent bel et bien de modèle aux négociateurs. L'analyse du mémorandum d'août 1950 nous oblige pourtant à atténuer sensiblement les revendications de paternité émises par Lagrange. Certains principes clés étaient déjà bien établis avant son arrivée à la conférence de Paris. Il était notamment clair que la Cour ne serait pas une juridiction internationale dans la lignée de celle de La Haye et qu'elle serait appelée avant tout à juger la légalité des actes des organes de la communauté du charbon et de l'acier et non leur opportunité.

La transposition de ces principes généraux en articles précis fut néanmoins l'œuvre de Lagrange et elle suscita bien des émois. L'ancien conseiller d'État évoqua la réaction «de surprise et de stupéfaction» ${ }^{83}$ que les clauses qu'il proposait suscitèrent parmi les autres délégations. Sur ce point, sa mémoire est bonne: on assista en effet à une véritable levée de boucliers. Dans le projet de traité du 8 novembre, Lagrange atrophia en effet considérablement les compétences de la Cour et ignora certains principes fixés d'un commun accord dans le mémorandum du 10 août. ${ }^{84}$ Il n'était ainsi plus question d'un recours en cas de carence de la Haute Autorité, ce qui fit bondir les Luxembourgeois, qui craignaient que celle-ci ne développe «une tendance naturelle à délaisser les petits pays tandis qu'elle serait portée à venir au devant des desiderata des grands pays». ${ }^{85}$ Avaient également disparu la possibilité de recours contre une décision de la Haute Autorité susceptible de provoquer des troubles graves et persistants dans l'économie d'un

\footnotetext{
81. Aucune nouveauté relative à la Cour ne fut introduite dans le «Mémorandum sur les institutions et les dispositions économiques et sociales permanentes du Plan Schuman» du 28 septembre 1950.

82. Interview de M. Lagrange, op.cit.

83. M. LAGRANGE, La Cour de Justice des communautés ..., op.cit., p.128.

84. ANL/AE, dos.11349, Plan Schuman. Projet de traité, 08.11.1950.

85. ANL/AE, dos.11385, Les institutions de la Communauté.
} 
pays-membre et la procédure de médiation qui devait suivre l'arrêt de la Cour. Le droit d'appel contre les sanctions prises par la Haute Autorité n'était plus prévu. L'article 26 en particulier fut l'objet de bien des critiques. Directement inspiré du droit français, il prévoyait un recours en annulation «pour incompétence, violation des formes substantielles, abus de droit ou détournement de pouvoir». ${ }^{86}$ Ses détracteurs lui reprochèrent de substituer à la notion d'excès de pouvoir le concept d'abus de droit et de détournement de pouvoir, une invention du Conseil d'État français peu connue dans les autres pays. Ils dénoncèrent aussi la suppression du recours en annulation pour violation du traité.

Déplorant amèrement ces insuffisances, Muûls se demanda même s'il ne valait pas mieux «renoncer à l'institution d'une Cour plutôt que d'en élaborer un simulacre». ${ }^{87}$ Hallstein souligna que la Cour ne pouvait pas être pensée selon le droit administratif d'un seul et unique État de la communauté. Il insista sur la nécessité de créer un organe judiciaire relevant pleinement du principe supranational et capable de développer un droit européen commun. ${ }^{88}$ Dans le projet de traité du 8 novembre lui-même et lors de discussions subséquentes, la délégation française défendit sa position, argumentant que cette réduction des pouvoirs de la Cour était indispensable pour concilier, d'une part le souci de maintenir l'action des organes communautaires dans les limites du droit et, d'autre part, la nécessité tout aussi vitale de ne pas entraver l'action de la Haute Autorité dans un domaine où les considérations économiques, politiques et sociales exigeaient une constante appréciation des circonstances de fait ou d'opportunité échappant normalement à la compétence d'un juge. ${ }^{89}$ Uri rappela aussi qu'aucune décision de la Haute Autorité ne serait adoptée sans consultation préalable du Conseil des ministre et du Comité consultatif et que, pour les mesures les plus importantes, un avis conforme de l'organe intergouvernemental serait requis. La réponse du représentant du Luxembourg traduit parfaitement l'état d'esprit de nombreux négociateurs:

«si l'action conjointe avec le Conseil diminue les risques, elle ne les élimine pas. Si l'on demande de faire confiance à la Haute Autorité, il préfère accorder sa confiance à l'action des juges». ${ }^{90}$

On doit s'interroger sur les raisons qui incitèrent Lagrange à réduire les compétences de la Cour et, ce faisant, à revenir sur les accords laborieusement atteints en août. En l'absence de documents historiques permettant d'établir irrévocablement les circonstances de son intervention, on ne peut que spéculer. Lagrange a possiblement suivi des instructions plus ou moins directes de Monnet qui, une fois encore, aurait tenté de s'assurer que la Cour ne nuirait pas à la Haute Autorité. Sa décision de précipiter rue de Martignac un Lagrange qui, de son

86. Art.26, al.1 du projet de traité du 08.11.1950.

87. F. Muûls. Note concernant le projet de traité ..., 16.11.1950, op.cit.

88. PAAA, Abt.2, SFSP, dos.19, Kurzprotokoll über die deutsch-französische Juristensitzung im französischen Planungsamt am 21.11.1950.

89. ANL/AE, dos.11349, Plan Schuman. Projet de traité, 08.11.1950.

90. AMAE/B, Dos.gén. CECA 5216, Compte-rendu de la réunion du 10 novembre 1950. 
propre aveu, «ne sa[vait] pas de quoi il s'agi[ssai]t», ${ }^{91}$ traduit-elle son désir de limiter le rôle de la Cour européenne au seul contrôle de la légalité des actes normatifs et administratifs des institutions communautaires? Il n'est pas interdit de le penser.

Quelles que furent les instructions de Monnet, il semble néanmoins logique que Lagrange ait avant tout puisé dans le droit administratif français et dans sa longue expérience au Conseil d'État pour rédiger les dispositions relatives à la Cour, en particulier celles relatives au recours en annulation. Délibérément ou accidentellement, Lagrange a donc servi les intentions de Monnet. Ceci ne signifie cependant pas qu'il était opposé aux autres compétences de la Cour ni qu'il adopta toutes les positions de Monnet. Rappelons, par exemple, qu'il défendit le principe d'une Cour permanente alors que, sous l'influence de Lucien Hubert, Monnet proposait un organe ad hoc (mémorandum du 8 novembre). En fait, de l'avis des représentants belges, Lagrange fit preuve de «compréhension très large» à l'égard des revendications des autres délégations et «d'indépendance d'esprit à l'égard de Mr. Monnet». ${ }^{92}$

Enfin, il ne faut pas perdre de vue que les autres juristes français à la conférence, Gros et Hubert, étaient eux aussi partisans d'une stricte limitation voire d'une réduction des compétences de la Cour. ${ }^{93} \mathrm{Si}$, une fois encore, on se fie au témoignage d'un Muûls assez frustré par la méthode de travail imposée par Monnet, trois juristes français ont successivement contribué à la rédaction des dispositions relatives à la Cour, «sans que l'on puisse apparemment penser que l'un d'entre eux accepterait la paternité des textes élaborés». ${ }^{94}$ La tension qui régnait à l'automne 1950 au sein de l'équipe juridique française (en particulier entre Reuter et Lagrange $)^{95}$ a de toute évidence nuit à la cohérence des dispositions proposées dans le projet de traité du 8 novembre et à leur continuité avec les travaux antérieurs de la conférence.

Malgré les premières réactions défavorables, un terrain d'entente fut pourtant assez rapidement trouvé, sur base des propositions soumises par Paris et des remarques exprimées par les autres délégations. Des entretiens officieux permirent aux uns et aux autres d'expliquer leurs préoccupations puis de rapprocher leurs vues, préparant ainsi les réunions officielles des chefs de délégation. ${ }^{96}$ On négocia ardemment le recours pour violation de traité. Les Français craignaient par-dessus tout que, par ce biais, on ne donne aux juges le droit d'apprécier les faits ou les circonstances économiques, c'est-à-dire l'opportunité et pas seulement la légalité

91. J. MONNET, Mémoires, Fayard, Paris, 1976, p.413.

92. Note à M. le Ministre Muûls, 21.11.1950, op.cit.

93. AMAE/B, Dos.gén. CECA 5216, Entrevue de Mr. Muûls avec Mr. Gros, le 21 septembre; Hubert. Note confidentielle ..., 27.09.1950, op.cit.

94. Note à M. le Ministre Muûls, 21.11.1950, op.cit. Sans les nommer, Muûls parlait de «trois juristes du Quai d'Orsay».

95. Voir la contribution de Jérôme Wilson dans ce numéro.

96. Kurzprotokoll über die deutsch-französische Juristensitzung ..., 21.11.1950, op.cit; Note à M. le Ministre Muûls, 21.11.1950, op.cit. 
des décisions de la Haute Autorité. Après des discussions serrées,${ }^{97}$ il fut finalement convenu d'ajouter aux moyens susceptibles d'être invoqués à l'appui d'un recours en annulation (incompétence, violation des formes substantielles et détournement de pouvoir) la violation du traité, moyen élargi pour inclure aussi «toute règle de droit relative à son application (art. 33)». On inscrivit cependant aussi très clairement dans cet article 33 que l'examen de la Cour ne pourrait pas porter sur «l'appréciation de la situation découlant des faits ou circonstances économiques au vu de laquelle sont intervenues les dites décisions ou recommandations», sauf en cas de détournement de pouvoirs ou de violation manifeste du traité.

Cette formulation resta longtemps insuffisante pour le gouvernement luxembourgeois qui, pressé par son industrie sidérurgique, ${ }^{98}$ continua à s'opposer aux dispositions décrites ci-dessus. Il ne leva ses réserves que lorsqu'il obtint l'insertion dans le traité d'une clause de sauvegarde (art.37) prévoyant que

«lorsqu'un État membre estime que, dans un cas déterminé, une action ou un défaut d'action de la Haute Autorité est de nature à provoquer dans son économie des troubles fondamentaux et persistants, il peut saisir la Haute Autorité».

Celle-ci doit alors, après consultation du Conseil, prendre des mesures pour «mettre fin à cette situation tout en sauvegardant les intérêts essentiels de la Communauté». Des garanties juridictionnelles complétaient cette disposition: l'État qui s'estime victime de troubles fondamentaux peut saisir la Cour si la décision d'arbitrage de la Haute Autorité ne le satisfait pas ou si cette dernière refuse de reconnaître l'existence même de la situation. Par dérogation à l'article 33, la Cour est alors autorisée à apprécier toutes les circonstances de fait, même de nature économique. Considérant que cette disposition «pourra 'couvrir' tous les cas luxembourgeois en raison de l'importance énorme de la sidérurgie dans l'ensemble de l'économie du pays», les Luxembourgeois se rallièrent finalement à l'article 33. En dehors du fait qu'ils auraient été les seuls à «continuer la résistance», ils étaient conscients qu'il aurait été peu sage de donner à la Cour l'entièreté du pouvoir d'appréciation de l'activité de la Haute Autorité, vu la complexité des faits et des circonstances à apprécier. ${ }^{99}$

La question de savoir si la Cour devait ou non être accessible aux particuliers, c'est-à-dire aux entreprises, constitua un autre point de divergence important entre les délégations. Ceci ne doit en fait guère nous surprendre si l'on considère qu'en droit international, l'accès direct au prétoire n'est traditionnellement pas octroyé aux personnes privées mais réservé aux États. Le document de travail français du 24 juin prévoyait qu'un droit de recours leur serait ouvert mais uniquement contre les décisions et les recommandations de la Haute Autorité «les visant individuellement». Les représentants du Benelux (les Belges en particulier) s'opposèrent à cette possibilité et ce, pour différentes raisons. Ils craignaient

97. FJM, AMG 11/3/1, Note sur la compétence de la Cour de Justice (art.33 du projet de traité), 15.01.1951.

98. ANL/AE, dos.11349, GISL au gouvernement, Observations de la Sidérurgie au projet de traité daté du 8 novembre 1950.

99. ANL/AE, dos.11385, Les institutions de la Communauté. 
d'abord que la Cour ne soit rapidement submergée de recours. Ce droit était par ailleurs jugé inutile puisque les États prendraient immanquablement fait et cause pour leurs entreprises si elles subissaient un préjudice grave suite à une décision de la Haute Autorité. Enfin et surtout, une telle pratique aurait privé les tribunaux nationaux de leur compétence souveraine, ce qui, même s'il s'agit du secteur limité du charbon et de l'acier, soulèverait de «sérieuses difficultés de principe et de fait». ${ }^{100}$

La question ne pouvant être tranchée, elle fut réservée dans le mémorandum du 10 août 1950. Si Monnet lui-même souhaitait limiter le droit de recours aux seuls gouvernements, ${ }^{101}$ les Allemands poussaient très fermement vers une solution inverse. S'inspirant de leur Loi fondamentale, ils cherchaient à établir une cour de type fédéral, accessible aux entreprises et à leurs associations. Puisqu'il était admis, argumentaient-ils, que la Haute Autorité adresserait ses décisions et ses recommandations directement aux particuliers, il paraissait juridiquement incohérent de leur refuser la possibilité de saisir la Cour européenne. Un tribunal national ne pourrait pas, en effet, annuler une décision du collège supranational. ${ }^{102}$ Par ailleurs, une telle procédure favoriserait l'établissement d'une jurisprudence uniforme, ce qui importait énormément aux Allemands. ${ }^{103}$ La question fut laissée en suspens jusqu'au projet de traité du 8 novembre. Dans ce document, il fut stipulé que les entreprises pourraient introduire un recours, mais uniquement contre les décisions individuelles les concernant. ${ }^{104}$ Une entreprise n'aurait donc pas la possibilité de faire appel à la Cour européenne contre les décisions ou recommandations générales qui, sans la concerner directement, lui porteraient néanmoins préjudice sauf si elle estimait que ces décisions (ou recommandations) étaient «entachées de détournement de pouvoir» à son égard. Les décisions générales pourraient être attaquées en justice uniquement par les États membres ou le Conseil. L'accès des autres institutions de la Communauté (Haute Autorité, Conseil spécial des ministres et Assemblée commune) à la Cour ne posa quant à lui aucune difficulté.

Quelques mots enfin sur l'article 41, important surtout au regard de l'article 177 du traité de Rome (234 CE). Ces deux articles rendent la seule Cour de justice compétente pour résoudre, à titre préjudiciel, les questions soulevées devant les juridictions nationales. L'article 41 CECA se limite cependant à confier à la Cour une compétence exclusive uniquement en ce qui concerne «la validité des délibérations de la Haute Autorité et du Conseil» alors que le traité CEE autorise la Cour à statuer «sur l'interprétation du traité» ainsi que «sur la validité et

100. ANL/AE, dos.11372, Rapport du Comité des Juristes, 08.08.1950.

101. FJM, AMG 6/5/7, J. Van Helmont. Note sur l'entretien de M. Monnet avec M. Blaisse le 18 septembre 1950, 18.09.1950.

102. PAAA, Abt. 2, SFSP, dos.53, Übersicht über den Stand der Verhandlungen am 18.8.1950; AMAE/ B, Dos.gén. CECA 5216, Muûls, Plan Schuman. Note pour le CMCE, 24.08.1950.

103. CARAN, Commissariat Général du Plan, Série 81 AJ, dos.154, Ophüls. Premières observations de la délégation allemande, 02.08.1950.

104. Art.26, al.2 du projet de traité du 08.11.1950. 
l'interprétation des actes pris par les institutions de la Communauté». A posteriori, le caractère restrictif de l'article 41 surprend. Durant les négociations, les juristes en particulier allemands - avaient en effet plusieurs fois souligné l'importance d'assurer le développement d'une jurisprudence européenne uniforme et, à cette fin, de réserver à la Cour l'interprétation du traité. Depuis le projet de traité du 30 novembre 1950, une clause stipulait d'ailleurs clairement que la Cour avait «la mission d'assurer le respect du droit dans l'interprétation et l'application» du traité et des règlements d'exécution. ${ }^{105}$

L'idée des renvois préjudiciels fut, quant à elle, introduite plus tardivement. Lagrange la proposa en fait au comité des juristes, le 15 février 1951, «en vue d'éviter que les juridictions nationales se prononcent dans des questions mettant en cause la Communauté». ${ }^{106}$ Les dispositions de l'article 41 furent ajoutées pour compléter celles de l'article précédant qui répartit les compétences entre la cour européenne et les tribunaux nationaux dans les litiges découlant de fautes des agents de la communauté. La répartition fut basée sur le principe que la Cour européenne «connaît de tout ce qui concerne l'application du traité et ne connaît que de cela ${ }^{107}$. C'est pour garantir le respect de cette règle et ainsi éviter les conflits de juridiction que la procédure en renvoi préjudiciel a été introduite. Il semblerait que seul Muûls se soit opposé à cette disposition, en vain cependant puisque le texte soumis le 15 février fut repris, mot pour mot, dans le traité final. Le Belge reconnaissait certes qu'elle paraissait «satisfaisante en ce qui concerne le fonctionnement du Plan [Schuman]», mais il admettait difficilement la conséquence qui, selon lui, découlerait de la proposition de Lagrange: la suprématie de la Cour européenne sur les tribunaux nationaux en ce qui concerne l'interprétation du traité. ${ }^{108}$

Sa lecture de l'article 41 n'était pas correcte stricto sensu puisque, justement, l'article en question confère à la Cour le droit de statuer, à titre préjudiciel, non pas sur l'interprétation des actes de la Haute Autorité et du Conseil mais sur leur validité si cette dernière est contestée devant une juridiction nationale. On peut pourtant penser, à l'instar de Muûls, que, malgré cette formulation limitative, les auteurs de l'article 41 incluaient aussi un pouvoir d'interprétation du droit communautaire par la voie de questions préjudicielles. En 1990, dans l'arrêt CECA/Busseni, les juges européens reconnurent d'ailleurs que «la compétence conférée à la Cour par l'article 41 précité couvre non seulement l'appréciation de validité, mais aussi l'interprétation», cela au vu que (1) les articles 31 et 41 expriment «une double nécessité, celle d'assurer au mieux l'unité dans l'application du droit communautaire et celle d'établir à cette fin une coopération efficace entre

105. Art.24 quater du projet du 30.11.1950. Art.31 du traité de Paris.

106. AMAE/B, Dos.gén. CECA 5216, Note de M. Muûls, 16.02.1951.

107. Archives historiques des communautés européennes (Florence), Fonds Uri, dos.37/2, Lagrange. Élaboration de l'exposé des motifs, s.d.

108. L'alternative suggérée par Muûls prévoyait, en cas de contrariété de jugement, «l'intervention du Comité des ministres donnant en quelque sorte une interprétation législative du traité». Note de M. Muûls, 16.02.1951, op.cit. 
la Cour de justice et les juridictions nationales» et que (2) «il existe une connexité entre l'interprétation et l'appréciation de validité». ${ }^{109}$ L'état lacunaire des archives empêche malheureusement de préciser davantage tant les intentions et les motifs de Lagrange que les réactions de ses partenaires à l'égard de l'article 41. Il ne semble pas pourtant que les auteurs de cette disposition aient réalisé que la procédure du renvoi préjudiciel deviendrait un des plus puissants outils d'intégration.

À la fin du mois de novembre, la composition de la Cour, ses compétences et le mode de désignation des juges étaient donc déterminés. «Le système des voies de recours n'est pas parfait», commenta le chef de la délégation luxembourgeoise,

«toutefois nous ne croyons pas qu'en raison de son caractère technique, et en raison du fait que tous ces textes concrétisent des compromis péniblement obtenus, la question puisse être reconsidérée sur le plan ministériel». ${ }^{110}$

Et elle ne le fut pas.

\section{Conclusion}

L'étude attentive d'une variété d'archives relatives aux négociations du traité de Paris permet de jeter un regard nouveau sur la genèse de la première Cour européenne et de montrer les difficultés liées à l'établissement d'un contrôle judiciaire supranational. Quelques points méritent d'être relevés en conclusion de cet article.

D'abord, il apparaît clairement que les dispositions relatives à cette Cour résultèrent d'un compromis malaisé entre deux tendances. D'une part, certains protagonistes - Monnet en tête - reconnaissaient la nécessité de maintenir l'action des organes de la nouvelle communauté dans les limites du droit, mais redoutaient qu'une Cour trop puissante ne vienne entraver ou paralyser le travail de la Haute Autorité. Ils se montrèrent par conséquent très enclins à (dé)limiter avec une grande précision les compétences de l'organe judiciaire et veillèrent à lui retirer l'appréciation de la situation découlant des faits ou circonstances économiques déterminant les décisions de la Haute Autorité. Cette exigence, raisonnable si l'on considère que le collège supranational intervient avant tout dans un domaine économique, fut satisfaite à l'article 33 du traité. Face à ceux qui craignaient la mise en place d'un «gouvernement des juges» se regroupaient les négociateurs inquiets de la toute puissance de la Haute Autorité. Ils s'efforcèrent, pour leur part, de multiplier les garanties et de doter la Cour de moyens de contrôle étendus. Les délégués du Benelux - en particulier les Luxembourgeois et les Belges - formèrent le noyau dur de ce groupe. Ils obtinrent aussi satisfaction puisque, les limitations placées sur les pouvoirs de la Cour sont levées dans certains cas particuliers, le plus

109. Arrêt du 22 février 1990, CECA / Busseni (C-221/88, Rec._p._I-495) (cf. al.10-17).

110. ANL/AE, dos 11385, Les institutions de la communauté [mars 1951]. 
important étant celui prévu à l'article 37 (recours en cas troubles fondamentaux et persistants).

Classer dans ces deux seules catégories tous les participants au débat sur la Cour serait naturellement trop réducteur. De sérieuses nuances doivent notamment être apportées en ce qui concerne la délégation allemande et en particulier son chef, Walter Hallstein. De tous les négociateurs présents à Paris, c'est lui qui entrevit le plus distinctement le rôle exceptionnel que, par la production d'un droit communautaire, la Cour de Justice pourrait jouer dans l'affirmation d'une «conscience européenne». ${ }^{111}$ Puisqu'elle était chargée de l'interprétation du traité, cette institution s'apparentait à ses yeux autant à une cour constitutionnelle (Verfassungsgerichthof), similaire à la Cour Suprême des États-Unis qu'à une cour administrative. Plus d'une fois, durant et après les négociations, il fit référence à l'exemple américain et exprima l'espoir de voir les juges européens exercer une influence et un rôle analogues à leurs homologues d'outre-Atlantique. ${ }^{112}$ Les Allemands se firent ainsi les champions de l'organe judiciaire pas tant parce que, à l'instar des petits pays, ils cherchaient à contrôler par tous les moyens possibles la Haute Autorité, mais plutôt parce qu'il leur importait d'affirmer, au niveau européen, le concept d'État de droit. Outre sa permanence, ils exigèrent que la Cour fût directement accessible aux entreprises du charbon et de l'acier et à leurs associations, la transformant par cette «brèche considérable dans les principes traditionnels» ${ }^{113}$ en une sorte de tribunal fédéral.

Le 10 décembre 1952, lors de la séance inaugurale de la Cour CECA, Jean Monnet accueillit les sept magistrats avec des mots ambitieux: «Je salue en vous», leur dit-il, «non pas seulement la Cour de la Communauté européenne du charbon et de l'acier, mais aussi la perspective d'une Cour fédérale européenne suprême». ${ }^{114}$ Le caractère solennel de la séance se prêtait probablement bien aux envolées lyriques mais, si ces propos reflètent réellement sa pensée, on ne peut manquer d'être frappé par le chemin parcouru depuis mai 1950. Il est clair qu'initialement, l'inspirateur du Plan Schuman n'a pas du tout compris que ses objectifs d'intégration européenne pourraient être aussi servis par le droit et la voie judiciaire. Très protecteur de l'autorité supranationale qui formait le cœur de son projet visionnaire, il se méfiait viscéralement de tout autre organe qui aurait pu « «avoir le dernier mot» sur le fonctionnement de toute la communauté». ${ }^{115}$

Ses idées évoluèrent pourtant, probablement sous l'effet conjugué de différentes influences. Celle d'Hallstein notamment qui, à l'occasion de plusieurs rencontres, souligna l'importance d'établir une communauté de droit. Paul Reuter lui montra aussi comment la cour européenne, envisagée comme une sorte de Cour suprême,

111. Notizen. Hallstein à von Brentano, 08.08.1950, op.cit; cf. aussi Kurzprotokoll über die deutsch-französische Juristensitzung ..., 21.11.1950, op.cit.

112. Notizen. Hallstein à von Brentano, 08.08.1950, op.cit.; PAAA/Abt.2, 221-40, dos.749, Hallstein, Third US Resident Officers' Conference, 16.07.1951.

113. M.LAGRANGE, L'ordre juridique de la CECA ..., p.850.

114. Allocution de J. Monnet, 10.12.1950. http://www.ena.lu?lang=1\&doc=12104.

115. P.REUTER, La conception du pouvoir politique ..., op.cit., p.274. 
trouvait sa place au sein d'une future fédération européenne. ${ }^{116}$ Enfin, l'influence des conseillers américains s'exerça aussi mais de manière plus ambiguë. Leurs réflexions alimentèrent certes les craintes de Monnet, l'incitant rejeter certaines procédures de recours initialement prévues; mais elles le poussèrent aussi à reconnaître la nécessité d'établir un contrôle judiciaire par le biais d'une cour permanente dont le rôle pourrait être comparable à celui de la Cour suprême américaine. Malgré son revirement de position, Monnet resta prudent face à Cour. S'il accepta finalement «de s'en remettre à la sagesse des juges», ${ }^{117}$ il ne le fit qu'après s'être assuré que leurs pouvoirs ne mettraient pas en péril le travail de la Haute Autorité.

Personnage incontournable de l'histoire de la Cour de Justice Européenne, Maurice Lagrange (s')est souvent présenté comme une sorte d'homme providentiel qui arriva rue de Martignac avec dans son cartable le modèle parfait (le Conseil d'État) à appliquer à l'organisation du pouvoir judiciaire européen, cela au moment où les autres juristes, en manque d'imagination, étaient incapables de dépasser les principes du droit international. Les documents d'archives montrent clairement que cette vision relève davantage du mythe que de la réalité. La physionomie générale la Cour était déjà bien esquissée lorsque Lagrange vint prêter main forte aux juristes de la conférence de Paris, ce qui ne l'empêcha pas de faire des apports importants, inspirés par son expérience du droit administratif français. Le conseiller d'État n'arriva cependant pas avec toutes les solutions et il dut composer avec les exigences et idées des autres délégations.

Il est enfin frappant de constater à quel point la nature, la composition et les compétences de la Cour se révélèrent de manière progressive et empirique aux négociateurs. Il n'existait pas de plan d'ensemble ou de schéma théorique préétabli par les spécialistes du droit. Ce furent les exigences spécifiques, les peurs et le besoin de protéger les intérêts nationaux des États qui souvent façonnèrent les paramètres la Cour. Les impératifs politiques furent aussi déterminants que les facteurs juridiques. Les fondateurs de la première communauté européenne étaient certes conscients de créer une institution inédite, mais la majorité d'entre eux ne réalisa pas, à ce stade, le plein potentiel intégrationniste de la Cour. Ils la conçurent principalement comme la gardienne de la légalité dans l'application du traité. Elle constituait d'abord et avant tout une garantie au profit des États membres et des entreprises que la Haute Autorité n'outrepasserait pas les larges pouvoirs qui lui étaient confiés pour réaliser les buts du traité.

Paradoxalement pourtant, selon les conclusions que Lagrange tira de l'analyse des 28 arrêts rendus par les juges européens entre 1953 et 1958, le rôle de la Cour

116. Reuter porta un regard assez critique sur la Cour. Il dénonça les exigences des «pays de moyenne importance» qui conduisirent à la création d'une cour «extrêmement pesante» qui «va finalement "avoir le dernier mot" sur tout le fonctionnement de la Communauté». Il nota que «l'ensemble de la structure constitutionnelle du traité consacre d'une certaine manière un "gouvernement des juges"». (Idem.).

117. FJM, AMG 11/3/1, Note sur la compétence de la Cour de Justice (art.33 du projet de traité), 15.01.1951, p.69. 
dans le fonctionnement de la CECA fut «plus important qu'on ne l'avait prévu et, surtout, sensiblement différent». ${ }^{118}$ En effet, l'expérience montra que, plutôt que d'abuser de ses pouvoirs, la Haute Autorité, pour différentes raisons, fit preuve de timidité dans l'utilisation des moyens mis à sa disposition. Cette évolution eut des conséquences sur la Cour qui fut amenée à jouer un rôle plus large que celui assigné par le traité de Paris. Au lieu de se prononcer contre l'arbitraire et les excès de pouvoir de l'organe supranational, la Cour eut davantage besoin de défendre la communauté contre l'influence que les États et les puissants intérêts professionnels exercèrent pour imposer des solutions non conformes aux dispositions du traité. Ce phénomène explique peut-être pourquoi les auteurs des traités de Rome, tout en s'inspirant du modèle établi dans le cadre de la CECA, élargirent sensiblement les compétences de la Cour en 1957. Mais ceci est une autre histoire ...

118. M.LAGRANGE, Le rôle de la Cour de Justice des Communautés européennes tel qu'il se dégage de la jurisprudence, in: Droit social, 1(1961), pp.1-9. 


\section{Alles, was Sie über Europa wissen müssen}

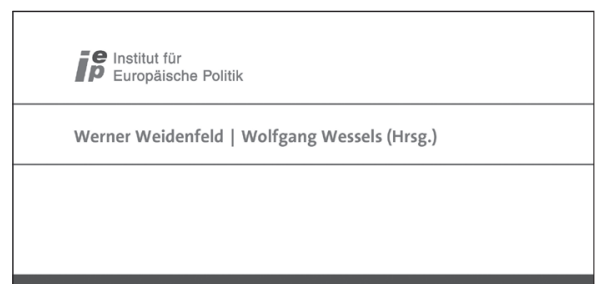

Jahrbuch der

Europäischen Integration 2008

Franco Algleri - David Allen . Juraj Alner - Franz-Lothar Altmann - Heinz-Jürgen Axt Esther Barbe - Peter Becker. Werner Becker - Stephan Bierling - Sandra Boldarinl - Barbara Hans-Georg Ehrhart . Heiko Furst - Anneli Ute Gabanyl : Armando Garcia Schmidt . Claus Glering - Daniel Gäler - Martin Großse Hüttmann - Christoph Cusy - Axel Huckstorf - Bernd Hütternann - Josef Janning : Mathias Jopp. Friedemann Kainer-Robert Kaiser - Iris Kempe

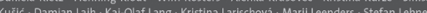
Christian lequesne - Rutger Lindahl . Heike Link - Ingo Linsenmann - Barbara Lippert Christian Lippert - Paul Liff - Florian Lütticken - Siegfried Magiera - Remi Maier-Rigaud Roman Maruhn . Andreas Maurer - Wim van Meurs. Almut Möller . Peter-Christia Müller-Graff. Florian Neuhann - Matthias Niedobitek. Markus Palmen. Thomas Petersen Christine Pols ful . Nicholas Rees. Elifriede Regels berger - Michele Roth. Catharina Sorensen

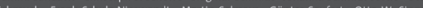
Serth Gahy Umbach. Ginther Unser - Werner Weidenfeld. Wolfgang Wessels . Klaus Winkles Wichard Woyke. Peter A. Zervakis

Nomos
Das Jahrbuch der Europäischen Integration des Instituts für Europäische Politik (iep) dokumentiert und bilanziert seit 1980 zeitnah und detailliert den europäischen Integrationsprozess.

Das „Jahrbuch der Europäischen Integration 2008" führt diese Tradition fort. In über 70 Beiträgen zeichnen die Autorinnen und Autoren in ihren jeweiligen Forschungsschwerpunkten die europapolitischen Ereignisse des Berichtszeitraums 2007/2008 nach und informieren über die Arbeit der europäischen Institutionen, die Entwicklung der einzelnen Politikbereiche der EU, Europas Rolle in der Welt und die Europapolitik in den Mitgliedstaaten und Kandidatenländern.

\section{Stimmen zur Vorauflage:} "anregend und informativ"

Curt Gasteyger, Neue Zürcher Zeitung 4.7.2008

"Das Buch ist wieder unverzichtbar." Prof. Dr. Erich Röper, Verwaltungsrundschau 5/08

\section{Jahrbuch der Europäischen Integration 2008}

Herausgegeben von Prof. Dr. Dr.

h.c. Werner Weidenfeld und Prof.

Dr. Wolfgang Wessels

2008, ca. 540 S., brosch., ca. 49,- $€$,

ISBN 978-3-8329-4162-8

Erscheint Dezember 2008 